\title{
A fiber tip refractive index sensor using FIB-milled gold-coated singlemode-multimode-singlemode structure
}

\author{
Ming Ding* ${ }^{\mathrm{a}, \mathrm{b}}$, Pengfei Wang ${ }^{\mathrm{d}}$, Junlong Wang ${ }^{\mathrm{c}}$, Heng Yuan ${ }^{\mathrm{a}}$,Gilberto Brambilla ${ }^{\mathrm{b}}$ \\ ${ }^{\text {a } S c h o o l ~ o f ~ I n s t r u m e n t ~ S c i e n c e ~ a n d ~ O p t o-E l e c t r o n i c s ~ E n g i n e e r i n g, ~ B e i h a n g ~ U n i v e r s i t y, ~ B e i j i n g, ~}$ \\ China 100191; \\ ${ }^{\mathrm{b}}$ Optoelectronics Research Centre, University of Southampton, Highfield, Southampton, UK \\ SO171BJ; \\ ${ }^{c}$ Beijing Aerospace Times Optical-electronic technology Co., No.1 Fengying East Road, Haidian \\ district, Beijing, China 100094; \\ ${ }^{\mathrm{d}}$ Photonics Research Center, School of Electronic and Communications Engineering, Dublin \\ Institute of Technology, Kevin Street, Dublin, Ireland Dublin 8.
}

\begin{abstract}
A compact fiber tip refractive index sensor using FIB-milled gold-coated singlemode-multimode-singlemode structure is demonstrated. Focused ion beam (FIB) is exploited to cut the fiber tip to obtain a flat end-face and then a layer of gold is coated on the tip surface, to increase its reflection. An average sensitivity of $265 \mathrm{~nm} / \mathrm{RIU}$ is obtained experimentally with a $~ 2.94 \mu \mathrm{m}$ diameter singlemode-multimode-singlemode fiber tip (SMST). Because of several advantages, including compactness, ease of fabrication, linear response, high sensitivity, easy connection with other fiberized optical components and low cost, this refractive index sensor could find various applications in chemical and biological area.
\end{abstract}

Keywords: refractive index sensor, focused ion beam, singlemode-multimode-singlemode

\section{INTRODUCTION}

Fiber optic sensors represent a technology base that can be applied to a multitude of sensing applications. Most physical properties can be sensed optically with fibers. They offer numerous advantages over conventional sensors due to their immunity to electromagnetic interference, resistance to erosion, small size, high sensitivity and capability of remote sensing. In the past years, several types of optical fiber sensors used to measure refractive index (RI) have been developed. The most common approaches are refractometers based on a fiber Bragg gratings (FBGs) [1], long period grating (LPGs) [2], surface plasmon [3], Fabry-Perot interferometer [4], and microfiber coil resonator [5]. However most techniques suffer from drawbacks: for example, grating (FBGs and LPGs) based sensors often require special fibers, hydrogen loading, a cumbersome grating writing equipment working with toxic gases and post fabrication treatments.

In 2006, a singlemode-multimode-singlemode (SMS) fiber based sensor utilizing multimode interference in the multimode fiber (MMF) core section has been proposed [6]. Concurrently, significant effort has been devoted to develop tapered fiber devices because of a range of advantages such as a large evanescent field, strong confinement, smart footprint, compact physical size and fast response [7]; hence tapering offers the potential both to improve the performance and to reduce the physical size of the SMS structure based fiber sensor mentioned above. Wang et al. demonstrated a singlemode-tapered multimode-singlemode (STMS) fiber sensor with $1900 \mathrm{~nm} / \mathrm{RIU}$ sensitivity at a RI 1.44 [8]. The STMS sensor consists of an input singlemode fiber (SMF), a tapered multimode fiber (MMF) section, and an output SMF. Within the tapered MMF section, the excited modes of $\mathrm{LP}_{0 \mathrm{~m}}$ in the MMF core is partly coupled to the high-order cladding modes at the beginning of the fiber taper region, and this increases the fraction of power in the evanescent wave within the region of the MMF cladding. However, given that a long portion of an MMF needs to be exposed to the surrounding medium in order to achieve a large refractive index change, the device results to be several centimeters long. 


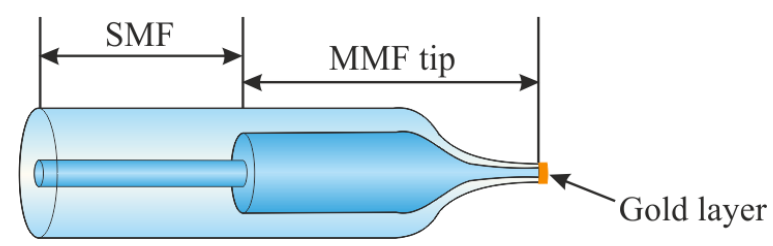

Figure 1 Schematic of gold-coated SMST.

In this paper, a compact singlemode-multimode-singlemode fiber tip (SMST) refractive index sensor is presented (Figure 1). The structure consists of an input/output SMF, an MMF half taper section and a gold film at the end of the tip as a reflection mirror. In both the conical transition region and tip sections, the effective index of the guided mode (thus multimode interference) is affected by the external medium RI, the MMF tip can be potentially used as a highly sensitive refractometer. Due to the small size, the SMST has a high spatial resolution and could be inserted into narrow area.

\section{GOLD-COATED SMST REFRACTIVE INDEX SENSOR FABRICATION}

The SMS sample was manufactured from a $42 \mathrm{~mm}$ long Thorlabs AFS105/125Y step-index MMF, which was stripped, cleaved, and then spliced between two standard SMFs. $\mathrm{A} \mathrm{CO}_{2}$ laser (Synrad, Model 48-2KWL, with maximum power of $30 \mathrm{~W}$ at a wavelength of $10.6 \mu \mathrm{m}$ ) was used to fabricate the SMST. A ZnSe cylindrical lens with focal length of $254 \pm 0.5 \% \mathrm{~mm}$ focused the $\mathrm{CO}_{2}$ laser beam down to $\sim 150 \mu \mathrm{m}$. The SMS fiber device was fixed vertically on a support and a weight $(\sim 30 \mathrm{~g})$ was used to apply a constant tension to the end of the SMS. When the middle of the MMF section was exposed to the $\mathrm{CO}_{2}$ laser beam with an average output power of $15 \mathrm{~W}$, tapering occurred because of the tension applied to the fiber end by the weight. Figure 2 (a) presents the optical microscope image of the SMST. Light launched into the SMST is reflected by the tip end. However, the reflectivity of this SMST is very weak and cannot be detected.

In order to increase reflectivity, the device tip was flat cut with the focus ion beam (FIB) system "Helios 600" (FEI Inc., Hillsboro, USA). A $50 \mathrm{~nm}$ layer of gold was deposited on SMST surface before FIB milling using an electron beam evaporator, to avoid charging during FIB milling. The Gallium ion beam accelerating voltage and current were $30.0 \mathrm{kV}$ and 93 pA, respectively. FIB beam sizes smaller than $30 \mathrm{~nm}$ can be easily obtained, thus tip end sizes can be controlled with a good degree of precision. Figure 2 (b) shows the SEM image of the SMST which was milled by FIB. The end of the SMST is measured to be $d \sim 2.94 \mu \mathrm{m}$.
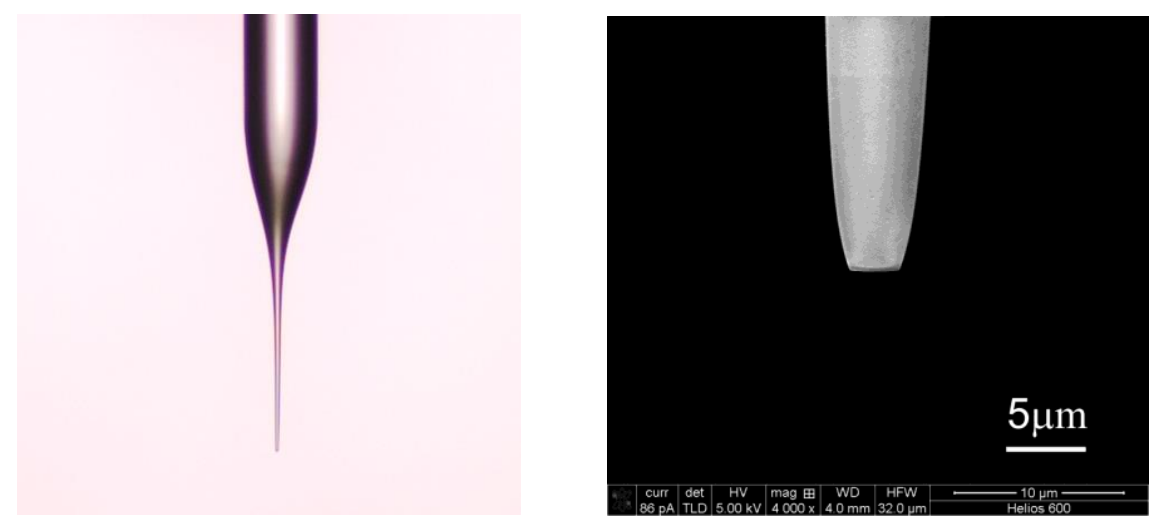

Figure 2 (a) Microscope image of SMST; (b) SEM image of FIB-milled SMST. The tip end size is $d \sim 2.94 \mu \mathrm{m}$.

After FIB milling, the gold layer was removed by gold-etching solvent which contains iodine and potassium iodine. Then another $40 \mathrm{~nm}$ layer of gold was coated on the milled SMST to increase the reflectivity further.

The reflection spectrum from $1350 \mathrm{~nm}$ to $1650 \mathrm{~nm}$ wavelength of FIB-milled gold-coated SMST in air is shown in Figure 3. Several dips appear in the spectrum, which corresponds to the different multimode interference. The reflectivity dips shows $>10 \mathrm{~dB}$ extinction ratio which is enough for sensing. Compared to the reflectivity in ref. [9], which was less than $-50 \mathrm{~dB}$, the reflectivity after FIB milling shows a significant improvement of $\sim 40 \mathrm{~dB}$. Therefore, FIB milling and gold coating can improve the reflection and therefore increase the signal to noise ratio. 


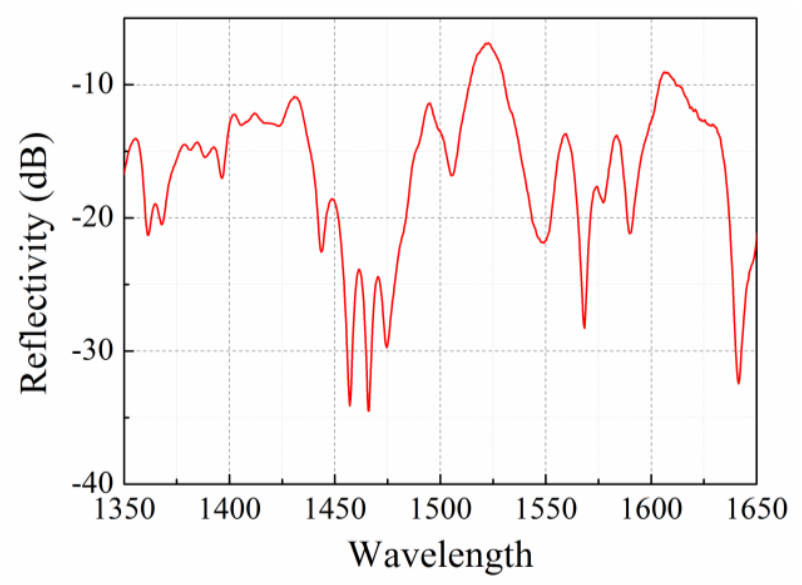

Figure 3 Reflectivity of gold-coated SMST in air after FIB milling.

\section{SMST REFRACTIVE INDEX SENSOR CHARACTERIZATION}

The refractive index sensing measurement was performed at a room temperature $\left(\sim 25^{\circ} \mathrm{C}\right)$ with a series of RI liquids (1.33 1.40 with an interval of 0.01, RI error \pm 0.0002$)$. The measurement set-up is shown in Figure 4. A supercontinuum (SC) source (Fianium Ltd, Hamble, U.K.) was used to deliver light over a rather broad range of wavelengths (450 nm$1800 \mathrm{~nm}$ ) with maximum pulse energy of $50 \mathrm{~nJ}$. The fiberized laser output was angle-cleaved to avoid back reflections from the optical components. The SMST was inserted into RI liquids. Reflection spectra were measured and recorded by an optical spectrum analyzer (OSA) (AQ6317, Yokogawa, Japan) via an optical circulator.

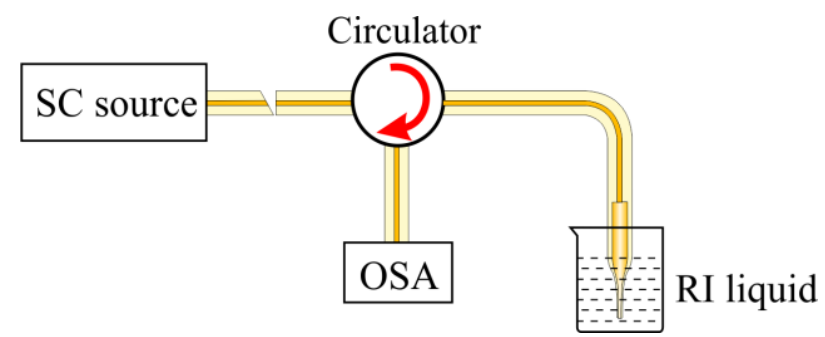

Figure 4 Experiment set-up for refractive index measurement.

The inset of Figure 5 shows the spectral shift of the peak at $\sim 1460 \mathrm{~nm}$ for refractive index increasing from 1.33 to 1.40. As the refractive index increases, the spectrum redshifts. The wavelength shift of the peak as a function of RI is plotted in Figure 5. An average sensitivity of $265 \mathrm{~nm} / \mathrm{RIU}$ (refractive index unit) is achieved, resulting in a resolvable index change of $3.77 \times 10^{-5}$ for a resolvable wavelength change of $0.01 \mathrm{~nm}$. Higher sensitivity can be achieved by implementing few improvements to the manufacturing technology, including: 1) writing a series of nanoslots or nanogratings on the fiber tip using FIB milling; 2) reducing the tip diameter and 3) using another peak in the reflection spectrum at longer wavelengths.

\section{CONCLUSION}

In summary, a compact refractometer which uses a FIB-milled and gold-coated SMST has been demonstrated. The refractometer has an average sensitivity of $265 \mathrm{~nm} / \mathrm{RIU}$ and a resolvable index change of $3.77 \times 10^{-5}$ for a resolvable wavelength change of $0.01 \mathrm{~nm}$, experimentally achieved with a $\sim 2.94 \mu \mathrm{m}$ diameter SMST. The sensitivity can be improved by optimizing the profile of the SMST. This fiber sensor offers several advantages, including compact size which would allow for good spatial resolution, but also ease of fabrication, linear response, high sensitivity, ease of interconnection with other fiberized optical components and low cost. The device is promising in various chemical and biological applications. 


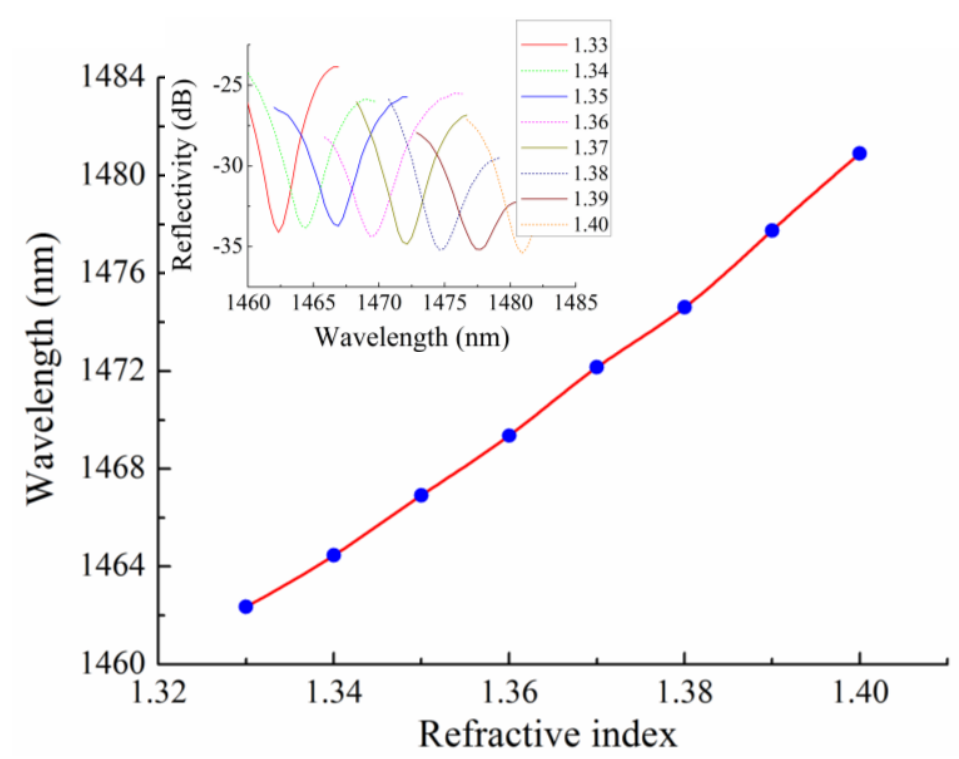

Figure 5 The wavelength shift of the peak as a function of RI. Inset: spectral shift of the peak at $\sim 1460 \mathrm{~nm}$ for refractive index increasing from 1.33 to 1.40 .

\section{ACKNOWLEDGMENTS}

GB gratefully acknowledges the Royal Society (London, UK) for his University Research Fellowship. P. Wang is funded by the Irish Research Council for Science, Engineering and Technology, co-funded by the Marie-Curie Actions under FP7.

\section{REFERENCES}

[1] Han, M., Guo, F. W., and Lu, Y. F., "Optical fiber refractometer based on cladding-mode Bragg grating," Opt. Lett., $35,399-401(2010)$

[2] Li, J. L., Zhang, W. G., Gao, S. C., Geng, P. C., Xue, X. L., Bai, Z. Y., and Liang, H., "Long-Period Fiber Grating Cascaded to an S Fiber Taper for Simultaneous Measurement of Temperature and Refractive Index," IEEE Photon. Technol. Lett., 25, 888-891 (2013)

[3] Trono, C., Baldini, F., Brenci, M., Chiavaioli, F. and Mugnaini, M., "Flow cell for strain- and temperaturecompensated refractive index measurements by means of cascaded optical fibre long period and Bragg gratings," Meas. Sci. and Technol., 22, 075204 (2011)

[4] Liao, C. R., Hu, T. Y. and Wang, D. N., "Optical fiber Fabry-Perot interferometer cavity fabricated by femtosecond laser micromachining and fusion splicing for refractive index sensing", Opt. Express, 20, 22813-22818 (2012)

[5] Xu, F.and Brambilla, G., "Demonstration of a refractometric sensor based on optical microfiber coil resonator," Appl. Phys. Lett., 92, 101126 (2008)

[6] Wang, Q. and Farrell, G., "All-fiber multimode-interference-based refractometer sensor: proposal and design," Opt. Lett., 31, 317-319 (2006)

[7] Brambilla, G., "Optical fibre nanowires and microwires: a review," J. Opt., 12, 043001 (2010)

[8] Wang, P., Brambilla, G., Ding, M., Semenova, Y., Wu, Q., and Farrell, G., "High-sensitivity, evanescent field refractometric sensor based on a tapered, multimode fiber interference," Opt. Lett., 36, 2233-2235 (2011)

[9] Wang, P., Ding, M., Bo, L., Semenova, Y., Wu, Q., Farrell, G., and Brambilla, G., "Fiber tip high temperature sensor based on multimode interference " Opt. Lett., vol. 38, 4617-4620 (2013) 